catch large prey.

After filming webs for

hundreds of hours, the researchers found no evidence that they trapped flying vertebrates such as birds, and in experiments the webs were unable to retain frogs or very large insects. These huge structures have unusually open architectures, and effectively provide large surfaces to catch semi-aquatic flying insects. Mass emergences of such creatures might provide an amount of food equivalent to a larger prey.

\section{J. Arachnol. 39, 287-295 (2011) Doctoring the
beats}

If you have endured much 1980s pop music, you might agree that drum machines (pictured) steal the soul from the beat. Their cold regularity is sometimes 'humanized' in the recording studio by the injection of random deviations from precise timing. But that's not good enough, according to Holger Hennig of the Max Planck Institute for Dynamics and Self-Organization in Göttingen, Germany, and his team, who analysed recordings of human drummers and singers performing simple rhythmic patterns.

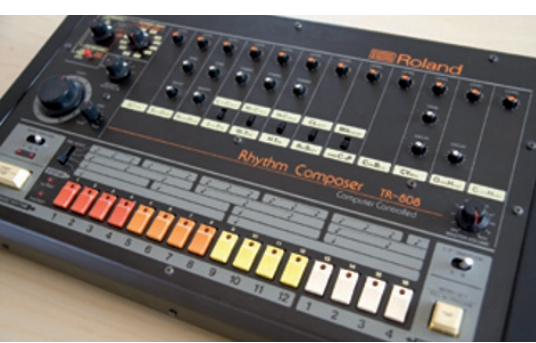

The researchers find that the rhythmic imperfections of human performers are not statistically independent, but show long-term correlations: one deviation can influence others for tens of seconds afterwards. What's more, listeners prefer rhythms with these correlated defects over those with pure white-noise fluctuations.

PLoS ONE 6, e26457 (2011)

\section{Ancient whales were worm food}

Whale-bone-eating worms that lack guts and rely on bacterial symbionts to eat may have been as widespread millions of years ago as they are today. Nicholas Higgs at the University of Leeds, UK, and his team report that a 3-million-year-old fossil from Italy, thought to be a beaked whale, contains bore-holes left by a type of annelid worm. The authors

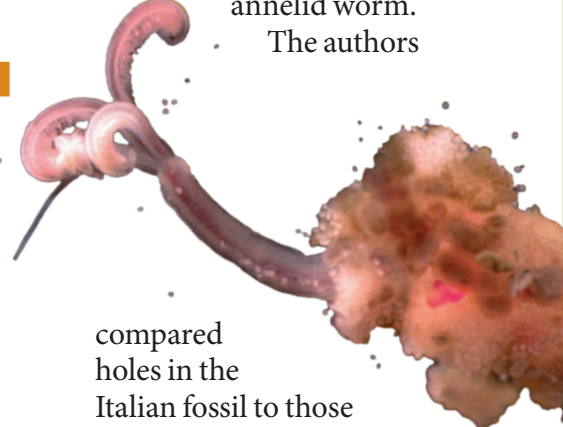

Italian fossil to those made by modern Osedax worms (pictured) in several whale bones. They found that the holes bore distinguishing characteristics of Osedaxmade canals. This is the second Osedax-eaten whale fossil to be identified - it follows a 30-million-year-old fossil from the northwestern United States - and the first discovery of Osedax in the Mediterranean, past or present, the authors say. Histor. Biol. http://dx.doi.org/1 0.1080/08912963.2011.6211 67 (2011)

\section{$3 D$ ripples in a 2D layer}

Two-dimensional single layers of carbon atoms known as graphene are thought to be stabilized by 'ripples' that deform the material into three dimensions. Andras Kis and his colleagues at the Swiss Federal Institute of Technology Lausanne (EPFL) report similar structures in molybdenum disulphide.

In the first transmission electron microscopy project to study single layers of $\mathrm{MoS}_{2}$, the

COMMUNITY CHOICE

The most viewed papers in science

\title{
How Rover got his wrinkly skin
}

\section{HIGHLY READ \\ on www.plos.org in October}

An analysis of several dozen dog breeds has yielded a genetic catalogue that can be used to search for information about complex canine traits and diseases.

Matthew Webster of Uppsala University in Sweden and his colleagues looked for single-base changes in the DNA of 509 dogs representing 46 breeds. The dogs spanned a wide range of shapes, colours and sizes, from tiny chihuahuas to huge Newfoundlands.

The researchers found 44 regions of the genome that varied widely between some breeds, and hundreds of regions that varied very little within breeds, suggesting that years of breeding have selected for traits based in these regions. Closer examination of such regions revealed various gene associations, including one that causes leg deformities in dachshunds and another for wrinkled skins in Shar Peis. PLoS Genet. 7, e1002316 (2011)

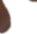

authors show that although the layers have long-range crystalline order, ripples of up to 1 nanometre in height can be observed. The team also identifies differences in the diffraction patterns of singleand multi-layer $\mathrm{MoS}_{2}$, which could be used to identify single layers. This is the first electron-microscopy study of large $\mathrm{MoS}_{2}$ sheets known to be just one layer thick. Moreover, $\mathrm{MoS}_{2}$ is only the second material in which such ripples have been confirmed, say the authors.

Nano Lett. http://dx.doi. org/10.1021/nl2022288 (2011)

\section{CANCER BIOLOGY}

\section{Fat fuels abdominal cancers}

Fat-storing cells called adipocytes are major players in the spread of ovarian cancer to a fatty abdominal organ called the omentum.

Using cultured human adipocytes and ovarian cancer cells, Ernst Lengyel of the University of Chicago in Illinois and his colleagues found that hormonal signals from the omentum's adipocytes attract cancer cells. This could explain why ovarian cancers often metastasize to the omentum. In culture, adipocytes transfer lipids to ovarian cancer cells, which use them as an energy source and grow rapidly.

Blocking the fat-binding protein FABP4 reduces both lipid transfer to cancer cells and the number of invasive cancer cells. This suggests that FABP 4 could be a target for fighting metastasizing abdominal tumours.

Nature Med. http://dx.doi. org/10.1038/nm.2492 (2011)

\section{CORRECTION \\ The bird pictured in the research highlight "Tools don't make for brainy birds" (Nature 478, 431; 2011) was incorrectly identified as a small tree finch owing to an error from the picture's source. It is actually a vegetarian finch.}

\section{$\rightarrow$ NATURE.COM}

For the latest research published by Naturevisit:

www.nature,com/latestresearch 